\title{
Multiphoton microscopy in defining liver function
}

Camilla A. Thorling

Darrell Crawford

Frank J. Burczynski

Xin Liu

Ian Liau

Michael S. Roberts

\section{SPIE.}




\title{
Multiphoton microscopy in defining liver function
}

\author{
Camilla A. Thorling, ${ }^{a, b}$ Darrell Crawford, ${ }^{b}$ Frank J. Burczynski, ${ }^{c}$ Xin Liu, ${ }^{b}$ lan Liau, ${ }^{d}$ and Michael S. Roberts ${ }^{a, b, *}$ \\ aUniversity of South Australia, School of Pharmacy and Biomedical Sciences, Adelaide, South Australia 5000, Australia \\ bUniversity of Queensland, School of Medicine, Woolloongabba, Queensland 4102, Australia \\ 'University of Manitoba, Faculty of Pharmacy, Winnipeg, Manitoba R3T 2N2, Canada \\ dNational Chiao Tung University, Department of Applied Chemistry and Institute of Molecular Science, Hsinchu 300, Taiwan
}

\begin{abstract}
Multiphoton microscopy is the preferred method when in vivo deep-tissue imaging is required. This review presents the application of multiphoton microscopy in defining liver function. In particular, multiphoton microscopy is useful in imaging intracellular events, such as mitochondrial depolarization and cellular metabolism in terms of $\mathrm{NAD}(\mathrm{P}) \mathrm{H}$ changes with fluorescence lifetime imaging microscopy. The morphology of hepatocytes can be visualized without exogenously administered fluorescent dyes by utilizing their autofluorescence and second harmonic generation signal of collagen, which is useful in diagnosing liver disease. More specific imaging, such as studying drug transport in normal and diseased livers are achievable, but require exogenously administered fluorescent dyes. If these techniques can be translated into clinical use to assess liver function, it would greatly improve early diagnosis of organ viability, fibrosis, and cancer. ๑ The Authors. Published by SPIE under a Creative Commons Attribution 3.0 Unported License. Distribution or reproduction of this work in whole or in part requires full attribution of the original publication, including its DOI. [DOI: 10.1117/1.JBO.19.9.090901]
\end{abstract}

Keywords: multiphoton microscopy; second harmonic generation; fluorescence lifetime imaging microscopy; fibrosis; hepatocellular carcinoma; mitochondria; drug transport; nanoparticles.

Paper 140088VR received Feb. 14, 2014; revised manuscript received Aug. 6, 2014; accepted for publication Aug. 19, 2014; published online Sep. 8, 2014.

\section{Introduction}

Most knowledge in the biological world has been gathered from studying images. ${ }^{1}$ Thus, a growing interest for live-tissue imaging has evolved. Fluorescence microscopy is highly sensitive and has good specificity, which makes it useful for studying the location and concentration of molecules and to view cellular events in real time. ${ }^{2,3}$ The first application of fluorescence microscopy in the liver occurred over 70 years ago. Since then it has provided vital information regarding normal and diseased liver function and morphology, ${ }^{4,5}$ vitamin A distribution, ${ }^{6}$ fat distribution, ${ }^{7}$ drug distribution, ${ }^{8,9}$ heterogeneity in drug distribution, ${ }^{10}$ and biliary function, ${ }^{11}$ to mention a few. In the last decade, these imaging techniques have been improved with confocal microscopy and nonlinear excitation microscopy, such as multiphoton microscopy (MPM). In conventional confocal microscopy, the intensity from the beam is approximately uniform above and below the focal plane, which results in the specimen generating fluorescence out of the focal plane that is rejected by the pinhole. This leads to the specimen being subjected to photobleaching and photodamage, affecting image quality and tissue health. ${ }^{2}$ MPM avoids this because a much smaller area of the specimen is being stimulated by the excitation light source and no out-of-focus light is generated, restricting photobleaching to the focal point only.,12 Due to the excitation wavelength of MPM, 700 to $1000 \mathrm{~nm}$ in the nearinfrared (NIR) range, at which the imaging penetration depth is maximized, tissue scattering and absorption are minimized. ${ }^{13,14}$ The most important application of MPM is to image the physiology, morphology, and cell-cell interactions of intact tissue of live animals with high resolution. Although

*Address all correspondence to: Michael S. Roberts, E-mail: m.roberts@uq .edu.au one limitation is that it cannot quantitatively study cellular function on a molecular level, ${ }^{15}$ MPM in combination with fluorescence lifetime imaging microscopy (FLIM) can, however, identify fluorophores with overlapping spectral properties and measure lifetimes, which are specific for the fluorophore and the environment surrounding it. ${ }^{16}$ Fluorescence lifetime is the reciprocal sum of the rate constants of all possible return paths for the electron from the excited state to the ground state. ${ }^{17}$ One application for FLIM in liver imaging is to study the levels of autofluorescent nicotinamide adenine dinucleotide (NADH), as a direct measure of the metabolic state of the cells. ${ }^{18}$ This review aims to highlight the use of MPM in defining liver function, in particular, assessing morphology, metabolic activity, and mitochondrial function, as well as diagnosing cancer and fibrosis and drug transport in healthy and diseased livers.

\section{Intracellular Imaging}

\subsection{Inflammation and Cell Death}

Neutrophils are considered responsible for the pathophysiologic changes in hepatic ischemia reperfusion (I/R) injury. The cascade starts with the initiation of Kupffer cells releasing cytokines, which induce the expression of chemokines and upregulation of endothelial cell adhesion molecules, which, in turn, mediate the adhesion and migration of neutrophils. Neutrophils release oxidants and proteases that directly injure hepatocytes and endothelial cells and might obstruct sinusoids, resulting in hepatic hypoperfusion (decreased blood flow). Neutrophils can be identified based on their different brightness level (fluorescent intensity) and distinct morphologic characteristics.

The study showed neutrophil recruitment in I/R injury using two-photon laser scanning microscopy. Low magnification showed that an overview of the liver and neutrophils could be measured more widely. High magnification revealed the 
presence or absence of perfusion in sinusoids and the neutrophil morphology at a single-cell level. The number of adherent neutrophils continued to increase for $4 \mathrm{~h}$ after reperfusion, whereas their crawling velocity reached a maximum $2 \mathrm{~h}$ after reperfusion. The dynamic change in size and fluorescent intensity of hepatocytes during I/R injury may morphologically show the process of hepatocyte death. ${ }^{19}$

MPM can also be used to visualize cell death by administering propidium iodide, which labels the nuclei of nonviable cells. ${ }^{20}$ Another way of imaging cell death without the administration of an exogenous marker is to measure the fluorescence intensity of cells. In a study of I/R injury in the rat liver, large dark areas in the I/R injury group were observed. The decrease in fluorescence was most likely due to a decrease in $\mathrm{NAD}(\mathrm{P}) \mathrm{H}$ (nicotinamide adenine dinucleotide + nicotinamide adenine dinucleotide phosphate) fluorescence, which occurs in cell death. The damage was found to be zonal, i.e., a heterogeneous spread in the liver damage caused by $\mathrm{I} / \mathrm{R}$ injury. The damage was located mainly in the midzonal region of the liver acinus. ${ }^{21}$ In paracetamol (acetaminophen) overdose, the most frequent cause of drug-induced liver failure in the United States and United Kingdom, hepatic apoptosis was found around the central vein. $^{22}$ Additionally, initial changes associated with paracetamol overdose included loss of mitochondrial membrane potential and basal membrane disruption. ${ }^{23}$

\subsection{Mitochondrial Depolarization}

Mitochondrial function is important for cell survival. ${ }^{20}$ In normal conditions, an electrochemical gradient of $H+$ ions is formed in the inner mitochondrial membrane as electrons are transferred through the respiratory chain. The energy of this electrochemical gradient drives ATP synthesis. ${ }^{24}$ Reactive oxygen species can trigger the opening of permeability transition pores in the inner membrane of the mitochondria, leading to mitochondrial permeability transition (MPT) ${ }^{25}$ MPT pores are highly conductive, nonselective megachannels in the mitochondrial membrane. ${ }^{26}$ MPT causes the mitochondrial membrane potential to collapse, i.e., the mitochondria will depolarize. ${ }^{26}$ The onset of MPT leads to (1) depletion of ATP causing necrotic cell killing (oncosis); (2) release of cytochrome c; and (3) outer membrane rupture. Severe ATP depletion leads to necrosis and moderate ATP depletion leads to apoptosis. ${ }^{27}$
Mitochondrial polarization can be detected in vivo by MPM and rhodamine 123 (Rh123) because Rh123 is taken up by polarized mitochondria. ${ }^{20}$ The fluorescence of Rh123 in healthy livers exhibits punctuate, bright, green fluorescence, denoting mitochondrial polarization [Fig. 1(a)]; however, in diseased livers, the fluorescence of Rh123 disappears and is characterized by dim diffuse staining [Fig. 1(b)]. . $^{28,29}$

MPT pore opening can be monitored by calcein acetoxymethyl ester (AM), which is cleaved by esterases in the cytosol to form green-fluorescing calcein-free acid. Mitochondria are normally impermeable to calcein, retaining calcein in the cytosol; however, after the onset of MPT, calcein enters the mitochondrial space through permeability transition pores. In sham operated mice, calcein loads the cytosol but not the mitochondria, outlining the mitochondria as dark voids. ${ }^{25}$

\subsubsection{Mitochondrial permeability transition studies in diseased livers}

MPT plays an important role in the pathogenesis of injury after $\mathrm{I} / \mathrm{R}$ injury and the opening of pores in the inner membrane causes MPT. ${ }^{28}$ Mitochondrial depolarization was detected in hepatocytes after massive hepatectomy ( $87 \%$ of the liver mass), but most of them were still viable, i.e., did not show propidium iodide staining. N-methyl-4-isoleucine cyclosporine (NIM811), a nonimmunosuppressive cyclosporin derivative, ${ }^{30}$ inhibits MPT in liver grafts and, thus, prevents mitochondrial depolarization. ${ }^{31}$ Ischemic preconditioning has also been shown to prevent the onset of MPT in small-for-size liver grafts. ${ }^{24}$

Additionally, the role of MPT in cholestatic liver injury in vivo using confocal/MPM was evaluated to see whether NIM811 prevents cholestatic cell death and liver fibrosis. In sham operated mice, calcein loads the cytosol but not the mitochondria, outlining the mitochondria as dark voids. In cholestasis, these voids disappear. ${ }^{25}$

\subsection{Cell Metabolism}

The ATP production in the mitochondria is governed by NADH, an important co-enzyme in cellular metabolism. ${ }^{21} \mathrm{NADH}$ is essential in the production of ATP. It can be free or bound to proteins, where binding is to mitochondrial membrane proteins, which mediates electron transfer from $\mathrm{NADH}$ to oxygen and results in the electrochemical gradient of $\mathrm{H}+$ ions, necessary

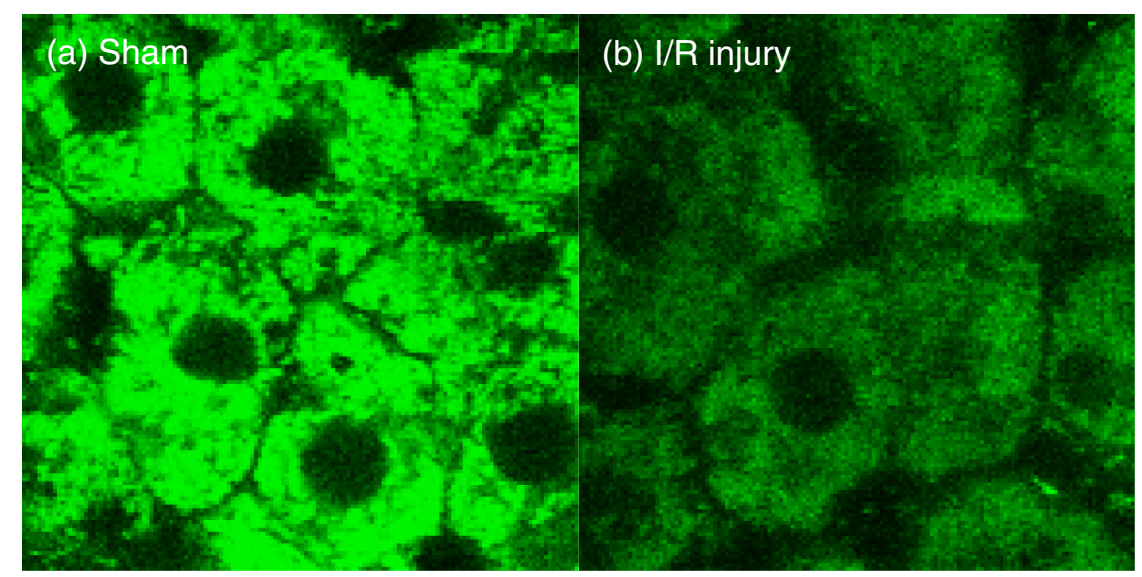

Fig. 1 Mitochondrial depolarization. (a) Sham operated mice show punctuation of rhodamine 123 (Rh123) fluorescence in all hepatocytes; in contrast, (b) mitochondria in many hepatocytes did not take up Rh123 in ischemia-reperfusion (I/R) injury. 
for ATP production in aerobic conditions. The free form of $\mathrm{NADH}$ is mainly located in the cytoplasm where glycolysis occurs, producing ATP in anaerobic conditions. Thus, free and protein-bound $\mathrm{NADH}$ are involved in the production of ATP, and changes in these molecules give insight into the metabolic state of a cell. FLIM is dependent on excited state reactions but independent of the concentration of the fluorophore, making it a useful tool in imaging NADH. ${ }^{21}$

MPM and FLIM have been used to follow disease progression in $\mathrm{I} / \mathrm{R}$ injury in anesthetized rats in vivo without the need for external markers. Results showed a significantly increased ratio of free/bound NAD(P)H in I/R injury, which was due to a statistical increase and decrease of free and bound $\mathrm{NAD}(\mathrm{P}) \mathrm{H}$, respectively [Fig. 2(a)]. The change can be seen as false colored images, where the increased ratio is indicated with a change from blue to yellow color. It is possible that the increased ratio is attributable to the Pasteur effect, where cellular respiration is shifted to glycolysis, producing ATP in anaerobic conditions. Glycolysis leads to lactic acid production, which was previously reported in pig liver during ischemia. ${ }^{21}$ Furthermore, the average lifetime $\left(\tau_{m}\right)$ of NAD(P)H/FAD (flavin adenine dinucleotide) (450 to $515 \mathrm{~nm}$ ) was significantly lower in I/R injury compared to sham [Fig. 2(b)]. Further examination revealed that the fast lifetime $\left(\tau_{1}\right)$ was significantly shorter in $\mathrm{I} / \mathrm{R}$ injury compared to sham, as shown in the decay curve in Fig. 2(c). The decay curve consists of two slopes, where the initial slope shows the fast lifetime $\left(\tau_{1}\right)$ and the second slope the long lifetime $\left(\tau_{2}\right)$. In Fig. 2(c), it is evident that the initial slope $\left(\tau_{1}\right)$ is sharper in I/R injury than in sham, indicating a shorter $\tau_{1}$. This study characterized changes in the metabolic state of hepatocytes after I/R injury, identifying a significantly decreased averaged weighted lifetime $\left(\tau_{m}\right)$ due to a decrease in the $\left(\tau_{1}\right)$ lifetime for $\mathrm{NAD}(\mathrm{P}) \mathrm{H}$ in the spectral channel 450 to $515 \mathrm{~nm}$. $\mathrm{NAD}(\mathrm{P}) \mathrm{H}$ may be recruited by similar proteins related to oxidative repair, thus causing the lifetime change. ${ }^{32}$

Investigating the fluorescence lifetime of $\mathrm{NAD}(\mathrm{P}) \mathrm{H}$ was also done in hepatoma cells. They found that 7-ketocholesterol, which is found in elevated amounts in patients with liver cancer and liver disease, shortened the average lifetime of $\mathrm{NAD}(\mathrm{P}) \mathrm{H}$, whereas cholesterol had no effect. The ratio of free/bound NAD $(\mathrm{P}) \mathrm{H}$ increased. These changes indicate the perturbation of energetic sources by 7-ketocholesterol. These results suggest a shift to glycolysis. ${ }^{33}$

MPM and FLIM have been shown to be useful in studying intracellular events, but how can this be translated into clinical practice in diagnosing liver disease?

\section{Imaging and Diagnosing Liver Disease}

\subsection{Fibrosis and Steatosis}

Liver fibrosis is a result of wound healing responses with resulting collagen accumulation. ${ }^{34}$ Chronic liver disease is often associated with extracellular matrix overproduction, such as collagen. Hepatic fibrosis is a result of an imbalance between fibrogenesis and fibrolysis responsible for the accumulation of fibrillar collagen. ${ }^{35,36}$ The abnormal increase in collagen causes derangement of liver architecture, impairment of intrahepatic blood flow, and subsequent progression and persistent liver dysfunction. ${ }^{36}$ Liver fibrosis leads to cirrhosis, liver failure, liver cancer, portal hypertension, and is particularly dangerous in chronic hepatitis B and $\mathrm{C}(\mathrm{HBV}$ and $\mathrm{HCV})$, where liver transplantation is frequently required. ${ }^{37}$

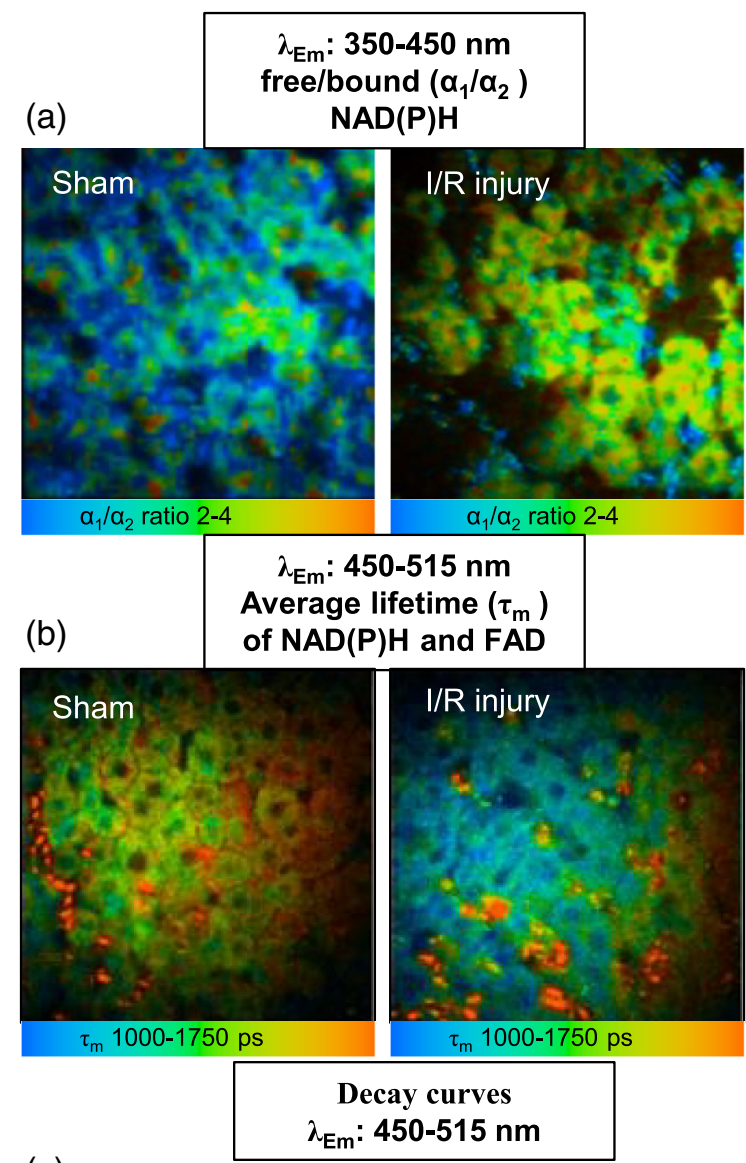

(c)

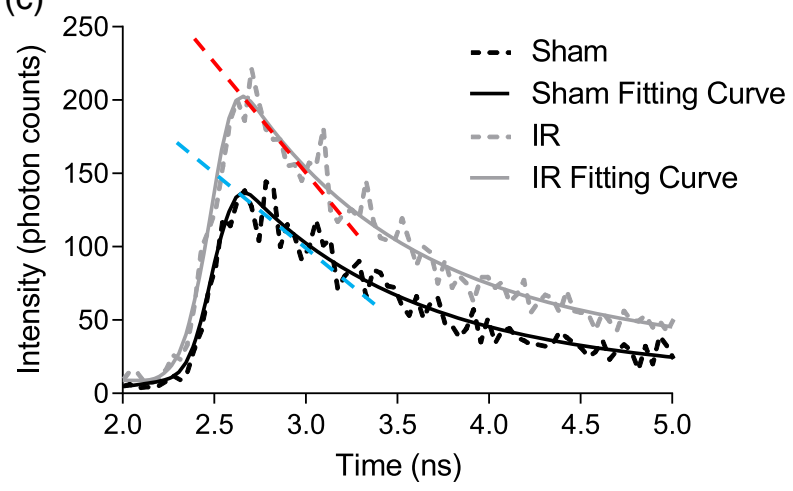

Fig. 2 Changes in the (a) redox ratio $(\alpha 1 / \alpha 2)$ of $\mathrm{NAD}(\mathrm{P}) \mathrm{H}$ and (b) and (c) average weighted lifetime $\left(\tau_{m}\right)$ of $\mathrm{NAD}(\mathrm{P}) \mathrm{H}$ and FAD within the rat liver after I/R injury. (a) Redox intravital images of sham (control) and $\mathrm{I} / \mathrm{R}$ injury in the liver measured within the spectral range of 350 to $450 \mathrm{~nm}$ [pseudo-color $\alpha 1 / \alpha 2$ range: 2 to 4 (blue to red)]. (b) Changes in $\tau_{m}$ of sham and $\mathrm{I} / \mathrm{R}$ injury in the liver. Fluorescence lifetime imaging microscopy data were measured within the spectral range [pseudocolor range (blue to red)] of 450 to $515 \mathrm{~nm}$ (1000 to $1750 \mathrm{ps}$ ) ( $n=4$ to 6). (c) Differences in decay curves for sham and I/R injury within the spectral range of 450 to $515 \mathrm{~nm}$. The dotted lines represent the data trace of the photons, whereas the solid lines represent the fit curve for the photons (black = sham, gray $=I / R$ injury). The red and blue dotted lines show the slope of the first component, representing the lifetime of $\tau_{1}$ in sham and I/R injury, respectively. ${ }^{21,32}$

Hepatic steatosis, or fatty liver, is characterized by an excessive accumulation of triglyceride within liver cells. Fatty liver might progress to steatohepatitis, cirrhosis, and even hepatocellular carcinoma. It is important to quantitate the amount of hepatic fat content in order to critically assess the progression 
of liver disease and efficacy of potential therapies against the disease. ${ }^{38}$

Fibrosis is treatable in its early stages; therefore, accurate staging is important for optimal treatment. ${ }^{39}$ Liver biopsy is the gold standard in tracking fibrosis, despite sampling error risk, ${ }^{40}$ but it is not ideal to perform serial liver biopsies in order to accurately determine disease progression or to monitor treatment effects. ${ }^{39}$ Therefore, noninvasive biomedical imaging techniques, such as ultrasonography, computed tomography, and magnetic resonance imaging, are important to detect liver disease. However, they do not have sufficient sensitivity, spatial resolution, and specificity to detect and stage liver disease at an early stage. This is a major limitation as it cannot predict the eventual timing of liver function loss, onset of portal hypertension, or other complications to determine proper therapeutic intervention..$^{34,40}$ Therefore, it is important to develop advanced optical imaging for noninvasive label-free quantitative assessment of early liver disease. ${ }^{40}$ Imaging has the potential to extract information over a long period of time and over a larger sampling area. ${ }^{39}$

\subsection{Multiphoton Microscopy}

MPM is the most popular application of fluorescence microscopy for imaging thick tissue and live animals, ${ }^{41}$ has the potential to take several virtual biopsies to obtain more of an overall view of the liver, ${ }^{42}$ providing dynamic information, ${ }^{43}$ and has the potential to quantify liver diseases, such as fibrosis. ${ }^{39}$

MPM is a term to describe a family of optical imaging modalities that employ optical signals produced through nonlinear (or multiphoton) processes. MPM comprises two-photon excitation fluorescence (TPEF), second harmonic generation (SHG), and coherent anti-Stokes Raman scattering (CARS). ${ }^{41}$

\subsubsection{Two-photon excitation fluorescence}

Compared to traditional histology and biomedical imaging, TPEF offers many advantages, such as three-dimensional optical sectioning, subcellular spatial resolution, deep penetration of thick specimens, and minimum destruction of samples. ${ }^{37}$ The signal arises from fiber elastin, NAD $(\mathrm{P}) \mathrm{H}$ and FAD that are related to cell metabolism, making TPEF suitable for imaging tissue and cell morphologies. ${ }^{44}$ TPEF images of a liver with fibrosis show a distinctly different morphology. Hepatocyte autofluorescence was similar in normal and fibrosis suggesting that the autofluorescence is not a sensitive indicator of liver fibrosis. ${ }^{45}$ However, the distribution of stellate cells is different. Using low magnification, large regions devoid of cellular structures become visible and stellate cells start to proliferate at the damage sites, consistent with histological images. ${ }^{46}$ These changes occur throughout the liver, including the surface. ${ }^{45}$ Stellate cells can provide a measure for possible photobleaching, because exposure to UV light for $10 \mathrm{~s}$ makes them disappear. They are also a morphological guide to where the blood flow runs due to their location in the Space of Disse. ${ }^{47}$ In order to detect and quantify levels of fibrosis by collagen, SHF is required.

\subsubsection{Second harmonic generation}

SHG is a coherent, nonabsorption process [Fig. 3(a)], distinguishing molecules or supramolecules that provide harmonic generation of light from fluorophores that emit fluorescence. ${ }^{36,48}$
It is ideal for tissue imaging since the specimen is not subjected to photodamage ${ }^{36}$ It does not require any fluorophore present in tissue; thus, signals are unaffected by dye concentration and photobleaching. ${ }^{34}$ Other advantages of SHG are as follows: (1) no deteriorated or photodamaged samples, (2) both SHG and TPEF can be used regardless of sample preparation, e.g., both frozen or paraffin-embedded tissue can be used, (3) easy and fast, and (4) no sampling bias as limits biopsies. ${ }^{35}$

SHG is used to measure highly ordered structures without central symmetry in tissue, such as collagen type $1 .^{34,36,44,48}$ Fibrillar collagen can generate a two-photon coherent process selectively collected and recorded at precisely the half-wavelength of excitation, which is characteristic of the SHG signature. ${ }^{35}$ SHG imaging clearly reveals the increase in fibrillar collagen between F1 (portal fibrosis) and F4 (cirrhosis). ${ }^{35}$ Gailhouste et al. demonstrated the use of TPEF and SHG in imaging liver fibrosis (Fig. 3). TPEF images were recorded at low magnification $(10 \times)$ and high magnification $(6 \times 0)$ without the use of any staining [Figs. 3(b) and 3(e)]. The excitation wavelength was set to $810 \mathrm{~nm}$ and a 405-nm bandpass filter was used. At low magnification, SHG was found mainly in the portal tracts [Fig. 3(c)], whereas at high magnification, the signal was highest inside the liver parenchyma [Fig. 3(f)]. Simultaneous TPEF and SHG imaging revealed the exact location of the SHG signal, showing the complementarity between these two modalities [Figs. 3(d) and 3(g)]. ${ }^{35}$

\subsubsection{Coherent anti-Stokes Raman scattering microscopy and multimodal imaging}

CARS is a type of nonlinear MPM technique, a label-free technique with high vibrational selectivity and sensitivity. CARS imaging is intrinsic and specific to molecular vibration, such as aliphatic $\mathrm{C}-\mathrm{H}$ bonds of lipids, and possesses chemical contrast without exogenous labeling. ${ }^{37,44,49,50}$ CARS microscopy has recently been integrated with Raman microspectroscopy to investigate intact normal and fatty liver tissues. ${ }^{38}$ Another way of imaging fibrosis is by combining TPEF, SHG, and CARS in an integrated imaging platform called multimodal nonlinear optical microscopy. ${ }^{40}$ Multimodal nonlinear optical microscopy has the potential to assess progression of liver disease in more detail than the various modules individually. These imaging modules make it possible to more specifically visualize the accumulation of collagen and fat on diseased liver tissues [Figs. 4(a) and 4(b)]. Rats were fed a diet deficient in methionine and choline for 8 [Fig. 4(b)] and 14 days [Fig. 4(c)]. As indicated in Fig. 4(b) (blue arrows), there were more small droplets in rats fed for a shorter time. The approach derives an advantage of multimodal imaging to simultaneously visualize different histopathological characteristics of intact tissues without using any stains [Fig. 4(c)]; integration of Raman spectroscopy into the imaging system further allows identification of these pathological features based on their spectral fingerprints [Fig. 4(d)]. For instance, the lines at $1070 \mathrm{~cm}^{-1}$ are assigned to $\mathrm{C}-\mathrm{C}$ stretching mode, those at $1259 \mathrm{~cm}^{-1}$ to $\mathrm{C}=\mathrm{C}-\mathrm{H}$ angular deformation, those at $1300 \mathrm{~cm}^{-1}$ to $\mathrm{CH}_{2}$ twisting mode, those at $1659 \mathrm{~cm}^{-1}$ to $\mathrm{C}=\mathrm{C}$ stretching mode, and those at $1740 \mathrm{~cm}^{-1}$ to $\mathrm{C}=\mathrm{O}$ stretching mode. The intense Raman feature at 2900 to $3000 \mathrm{~cm}^{-1}$ was associated with the stretching vibrations of $\mathrm{CH}_{2}$ and $\mathrm{CH}_{3}$ moieties. All these lines are associated with vibrations characteristic of triglyceride molecules and are consistent with the known knowledge that the hepatic fat comprises mainly triglyceride. This shows that multimodal MPM enables 


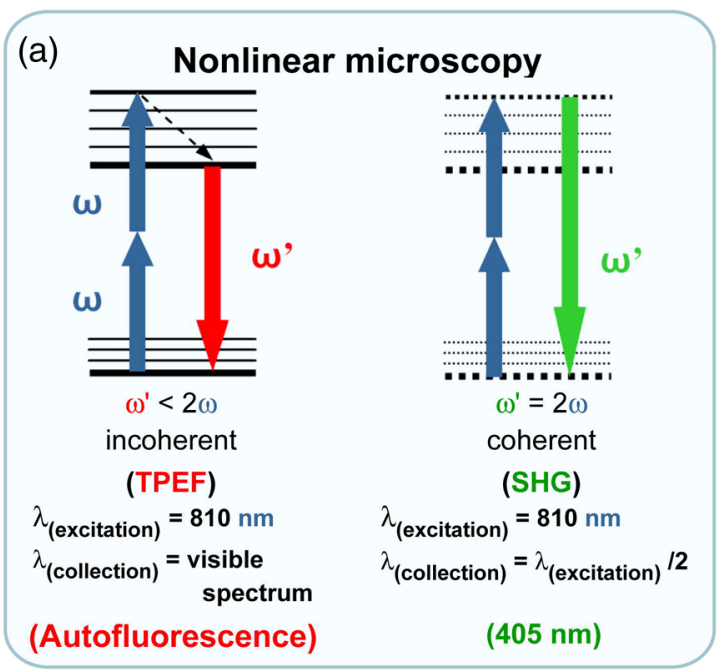

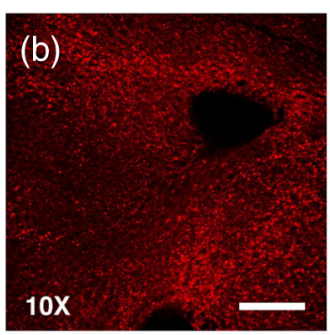
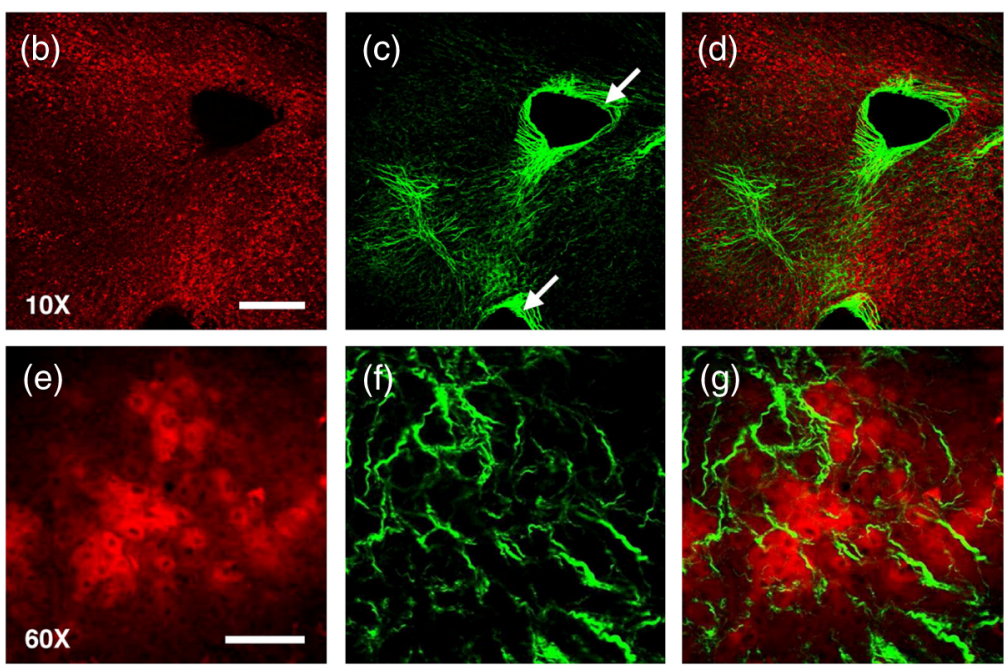

TPEF

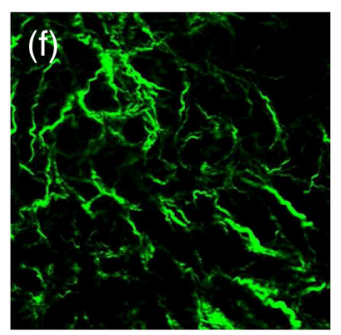

SHG

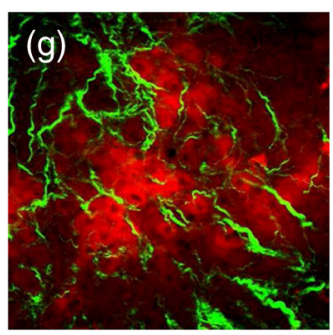

TPEF + SHG

Fig. 3 Two-photon excitation fluorescence (TPEF) and second harmonic generation (SHG) in human fibrotic tissue (reprinted from Ref. 35 with permission from Elsevier). (a) Principle of TPEF and SHG. (b) and (e) TPEF images of the liver at low and high magnification, respectively. (c) and (f) SHG signals collected at $405 \mathrm{~nm}$ at low and high magnification, respectively. (d) and (g) Combination of TPEF and SHG signals at low and high magnification, respectively.

(a)

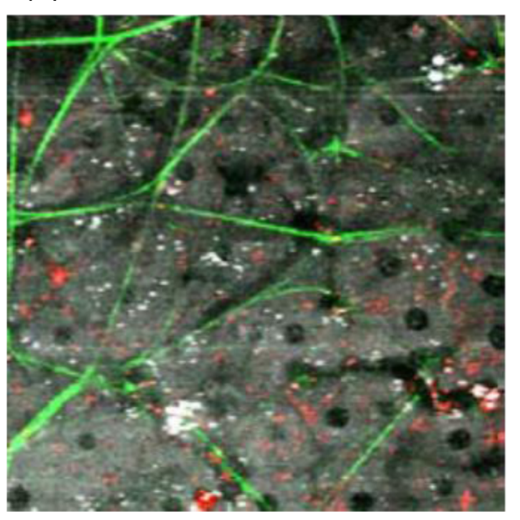

(c)

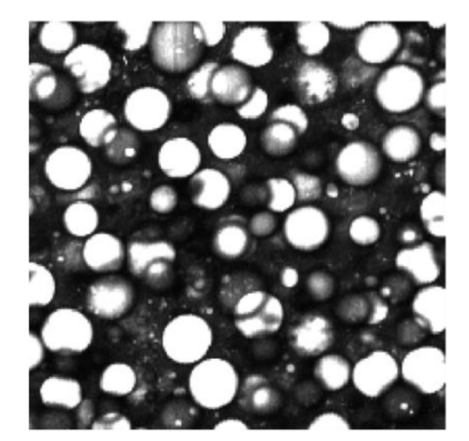

(b)

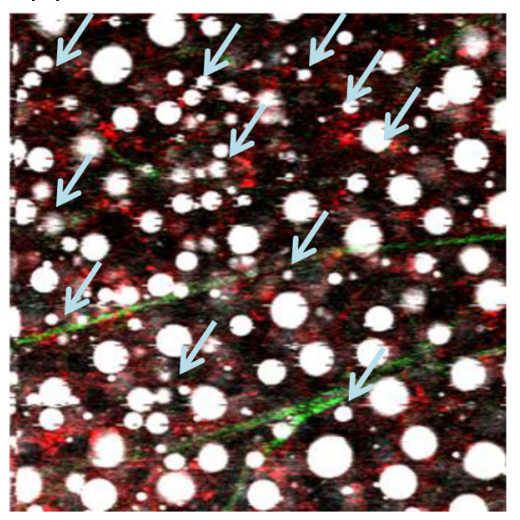

(d)

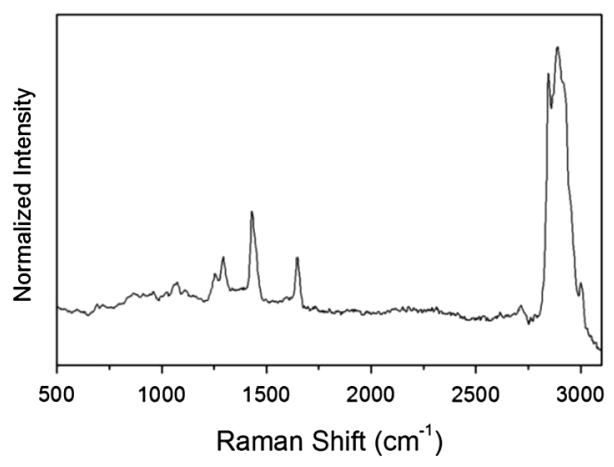

Fig. 4 Multimodal imaging. Multimodal images of intact normal (a) and fatty (b) liver tissues measured. Fatty change of the liver was induced by feeding the rat with a diet deficient in methionine and choline for eight days. The coherent anti-Stokes Raman scattering (CARS), SHG, and TPEF signals were coded in gray, green, and red, respectively. Scan area: $300 \times 300 \mu \mathrm{m}$; step size: $1 \mu \mathrm{m}$. (c) CARS image of intact fatty liver tissue obtained from a rat fed with a diet deficient in methionine and choline for 14 days (leftscan area: $200 \times 200 \mu \mathrm{m}$; step size: $0.5 \mu \mathrm{m}$ ). (d) Raman spectral characterization of hepatic fat (rightexcitation wavelength: $532 \mathrm{~nm}$ ). Analysis of the Raman spectrum shows that the hepatic fat comprises mainly triglyceride. 
visualization of both stromal and parenchymal tissues of liver with great contrast and definite spatial colocation with no need for any staining. The distinct pathological alteration associated with liver steatosis and fibrosis of varied severity is simultaneously identified from intact tissues without employing any labels.

\subsection{Fibrosis Quantification}

Liver fibrosis is reversible and accurate diagnosis is vital for accurate therapeutic managements. Histological scoring systems, like Scheuer, Ishak, or METAVIR, are qualitative or semiquantitative. ${ }^{48}$ For example, the METAVIR scoring system is used for the semiquantitative determination of liver fibrosis in biopsies. This system classifies liver fibrosis into five categories (grades 0 to 4 ) according to the morphological features of the collagen fibers. The key features are no fibrosis (0), portal fibrosis without septa (1), fibrosis with few septa (2), numerous septa without cirrhosis (3), and cirrhosis (4). ${ }^{51}$ Automatized analysis systems based on image segmentation, such as Fibroquant, Bioquant Nova Prime, and FibroXact, provide a continuum in fibrosis quantification, but the results are affected by a change in staining with protocols, time, and lack of internal reference. A simple scoring method based on SHG imaging has, therefore, been developed and correlates well with METAVIR assessment of fibrosis level. ${ }^{48}$

A standardized staging system, Fibro-C-Index, has been developed by combining SHG microscopy and adaptive quantification algorithm to identify collagen progression patterns specific to different fibrotic animal models. The system was also tested on human tissue, resulting in reduced time required for clinical diagnosis of liver fibrosis compared to traditional histology, by removing the tissue staining process and eliminating inter- and intraobserver discrepancies. ${ }^{34}$

TPEF can observe cellular and subcelluar details without the administration of exogenous contrast agents and provide realtime information about tissue architecture and cell morphology. One application is in liver cancers, for example, hepatocellular cancer (HCC). ${ }^{52-54}$

\subsection{Cancer}

The development and progression of HCC may be dependent on immune response, e.g., T-cell infiltration. HCC suppresses the immune system by overproducing Interleukin-10, decreasing the activity of dendritic cells, and activating regulatory $\mathrm{T}$ cells in tumor tissue. ${ }^{55} \mathrm{HCC}$ can be identified by the reduced intensity levels and longer fluorescence lifetime. ${ }^{56}$ Similarly, it has been suggested that the autofluorescence can be used to assist discrimination of $\mathrm{HCC}$ grade 1 due to significantly higher intensity in nontumor (NT) tissue. Therefore, one group sought to develop multiphoton autofluorescence (MAF) as an effective label-free tool in diagnosing HCC in ex vivo tissue. To characterize diseased tissue, they used quantitative parameters, such as nuclear number density (NND), nuclear-cytoplasmic ratio (NCR), and multiphoton autofluorescence intensity ratio. NND was done by calculating the number of nuclei in a field. NCR was done by randomly selecting five nuclei and determining their average MAF intensity. The cytoplasmic area is then determined by subtracting the nuclear area from the total image area. NCR can then be obtained from the ratio of the area occupied by the nuclear region over that of the cytoplasmic domain. The average MAF intensity of the imaged area was also determined. Results show higher NND and NCR in tumor tissue than in NT tissue. MAF is lower in tumor than nontumor tissue. ${ }^{57}$ Additionally, TPEF and SHG allow the respective identification of individual hepatocytes and collagen fibers in the HCC specimens, and the quantitative parameters of nuclear number density, the NCR, and autofluorescence intensity ratio enable clear discrimination of tumor from nontumor tissue. ${ }^{52}$ In addition to characterizing and diagnosing cancer, TPEF has also been used to investigate the antitumor effects of endostatin on colon carcinoma cells migrating to the liver. Antiangiogenic drugs are directed against components of the developing vasculature. Initiation of treatment is essential in the initial phase of tumor growth to optimize antiangiogenic therapy. Endostatin has shown to be an inhibitor of angiogenesis and has an antitumor effect. Intravital MPM was used to examine the fate of tumor cells in the liver. C26 cells were labeled with carboxyfluorescein succinyl ester. ${ }^{58}$

The usability of TPEF in diagnosing HCC has also been done in combination with FLIM. ${ }^{52,56}$ FLIM was used to assess molecular events in vivo in the whole-body using a tumor-specific NIR molecular probe. ${ }^{59}$

Most of these studies diagnosing liver fibrosis and cancer have been done by utilizing the cell's autofluorescent properties. This only reveals limited information and more specific imaging of liver function requires injection of various dyes. Fluorescent dyes not only are important in studying liver function, but can also be used to study transport in order to determine the dosage regimen for different diseases of the liver.

\section{Drug Transport}

The liver is the most important organ in the body for drug metabolism and excretion. ${ }^{9,60}$ When liver function is impaired due to ageing, nutrition, hepatitis, fibrosis, drugs, and even heart failure, drug clearance from the body could be affected, resulting in systemic and local toxicity ${ }^{61-64}$ or changed drug responses. Therefore, it is important to understand liver functional changes in a diseased status in terms of drug handling.

\subsection{In Vitro}

Dynamic analysis of uptake and metabolism of polycyclic aromatic hydrocarbons (PAHs) and their metabolites has the potential to provide novel insights into genotoxic and nongenotoxic mechanisms of cellular injury caused by PAHs. Benzo[a]pyrene $(\mathrm{BaP})$, one of the most important PAHs, occurs in tap water and has a potential carcinogenic metabolite activated by Cyp P450 $1 \mathrm{~A} 1,1 \mathrm{~A} 2$, and $1 \mathrm{~B} 1 .{ }^{65} \mathrm{BaP}$ is photosensitive and breaks down when exposed to UV light; therefore, conventional fluorescence microscopy limits the study of BaP. MPM, on the other hand, provides high detection sensitivity and minimal fluorophore excitation volume, minimizing photobleaching. ${ }^{66}$ This group investigated the uptake and subcellular partitioning of $\mathrm{BaP}$ in a rat-liver cell line (clone 9) and studied its effect on intracellular $\mathrm{Ca} 2+$ and cytoplasmic membranes using confocal and multiphoton microscopy. Results showed the simultaneous identification of eight metabolites in live cells at any single time that provide information about the cellular and molecular mechanisms of $\mathrm{BaP}$ and other PAHs. ${ }^{65,66}$

Fenestrations are present in the liver sinusoidal endothelial cells and their size and diameter can be altered by diabetes mellitus and old age. This could affect clearance of drugs, hepatic trafficking of lipoproteins, liver regeneration, and interactions between lymphocytes and hepatocytes. One study used TPEF to show how membrane rafts influence the formation of 
fenestrations in the liver and found that these are the final regulatory steps in their formation. Cells were stained by LAURDAN ( $\lambda_{\text {excitation }}=800 \mathrm{~nm}, \lambda_{\text {emission }}=425$ to $483 \mathrm{~nm}$ ), which undergoes a spectral blue-shift from 490 to $440 \mathrm{~nm}$ when in nonraft and raft regions, respectively. ${ }^{67}$

Cell line studies may have limited biological significance and do not allow a clear understanding of the spatial processes involved. ${ }^{68}$ Visualization of the solute concentration in time and space within organs would enable spatial pharmacokinetics to be better defined. ${ }^{47}$

\subsection{In Vivo}

TPEF has been used in vivo to visualize the biliary excretion of 6-carboxyfluorescein diacetate (6-CFDA) in normal ${ }^{69-71}$ and diseased $^{60,72}$ livers using a specially designed hepatic imaging chamber made of biocompatible titanium alloy. To limit the movement of liver tissue due to respiration and heartbeat, the edges of the imaging chamber were glued with the underlying liver tissue. ${ }^{69,71}$ The uptake of 6-CFDA and excretion of carboxyfluorescein $(\mathrm{CF})$ in bile in common bile duct ligation was quantitated by tracing the distribution of CF within hepatocyte and sinusoid compartments. Five small areas were circled for each compartment per image and the fluorescence intensity was calculated in each compartment. ${ }^{60,72}$ In this study, they presented, for the first time, kinetic changes of organic anions in two microscopic liver compartments (hepatocytes and sinusoids). In common bile duct ligation, there was a delayed clearance of $\mathrm{CF}$ out of the liver as well as higher intensities in the sinusoids. Data also showed downregulation of Oatp1, Mrp2, and Oatp2, and upregulation of Mrp4. By downregulating the uptake transporters (Oatp1 and 2) and upregulating Mrp4, more solute was kept in the circulation. ${ }^{60}$

In addition to CFDA and CF, the distribution of sodium fluorescein has been studied in normal ${ }^{47,73}$ and damaged livers ${ }^{32}$ in vivo with TPEF (Fig. 5). Fluorescein was quickly observed in the sinusoids following administration in the sham group. Uptake and clearance of fluorescein was found to be delayed in I/R injury, which were probably due to the impaired hepatic microcirculation and possible dysfunctional efflux transporter, respectively. ${ }^{32}$ TPEF proved to be a more sensitive method to measure liver function and to differentiate the impact of I/R injury than measuring bile and plasma concentrations of the compounds, because TPEF could specifically observe the compounds' disposition in the damaged lobes, whereas bile and plasma data provided information from the whole body only. ${ }^{32}$

Our group has also explored the role of P-glycoprotein (Pgp) in the disposition of Rh123 by administering an inhibitor for Pgp, cyclosporine A, in normal livers [Fig. 6(a)]. ${ }^{74}$ It was evident that the clearance of Rh123 was delayed and the excretion into bile was significantly decreased with cyclosporine A. ${ }^{74}$ In addition, the function of Pgp was also investigated in hepatic I/R injury using Rh123 as a probe [Fig. 6(b)]. Similarly, in I/R injury, there was also increased fluorescence intensity in the hepatocytes compared to sham, which was likely to be due to impaired Pgp function. This is in accordance with another study showing a significantly decreased biliary excretion rate and clearance of Rh123 in I/R injury at $24 \mathrm{~h}$ of reperfusion. ${ }^{75}$

These studies have evaluated the distribution of the dyes in a qualitative way by looking at the changes in probe substrate concentration-time curves in sinusoids, hepatocytes, and bile canaliculi. Another paper aimed to analyze data from TPEF using a compartmental model that describes hepatic transport processes. The model was able to quantify uptake, metabolism, distribution volume, and clearance and has been applied to sodium fluorescein and Rh123. The model is possible in sparse data situations and when intensive sampling is possible. A major advantage of this population approach is to include animals with an incomplete data set. This is the first study to estimate kinetic parameters of hepatic transport using intravital microscopy. The limitation is that a sinusoidal concentration-time curve was used as input function because the mass inflow rate into the sinusoids was missing, which is an approximate amount of fluorescent molecule in the sinusoidal compartment. In addition, efflux from cytosol to the sinusoidal space was neglected. ${ }^{68}$

Simultaneous imaging of diverse molecular processes would provide adequate in vivo characterization of diseases and an accurate prognosis. FLIM is not limited by the overlapping excitation and fluorescence spectra of many NIR dyes if they have different fluorescence lifetimes. ${ }^{76}$

\subsection{Fluorescence Lifetime Imaging Microscopy}

The possibility of resolving the distribution of two fluorophores given as a cocktail in mice using time-resolved diffuse optical imaging was assessed using FLIM. Results showed that the most fluorescent signal was detected in kidney, liver, and estrogen pellet regions. The signal from animal chow in stomach and

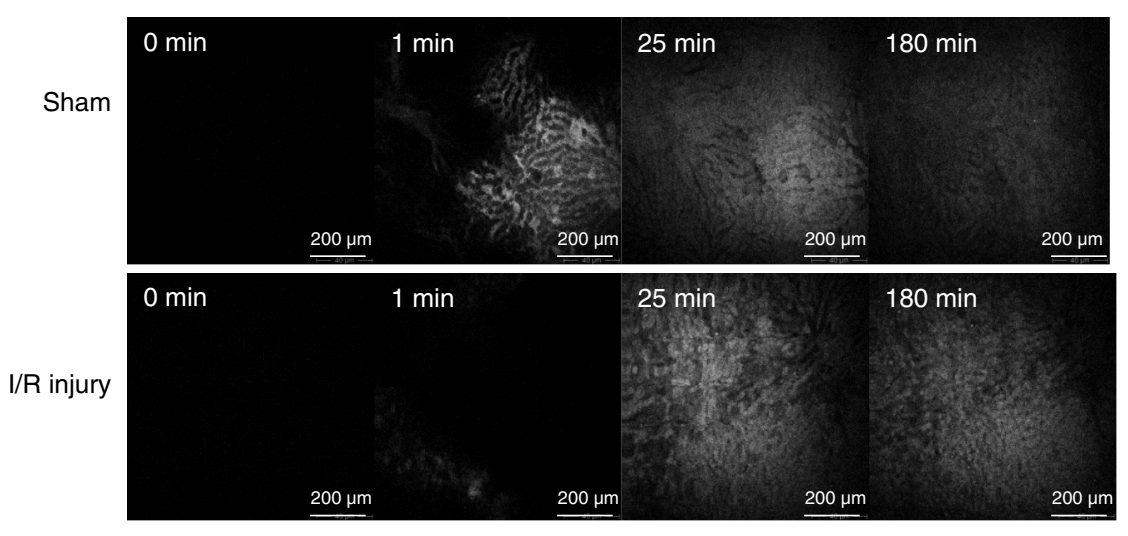

Fig. 5 Representative TPEF images of fluorescein's distribution in hepatic I/R injury compared to sham in rats. Images were recorded at various time-points, following ischemia insult or sham operation, using 10x magnification at $\lambda_{\text {Excitation/Emission }}$ of $920 / 350$ to $650 \mathrm{~nm}(n=3)$. 

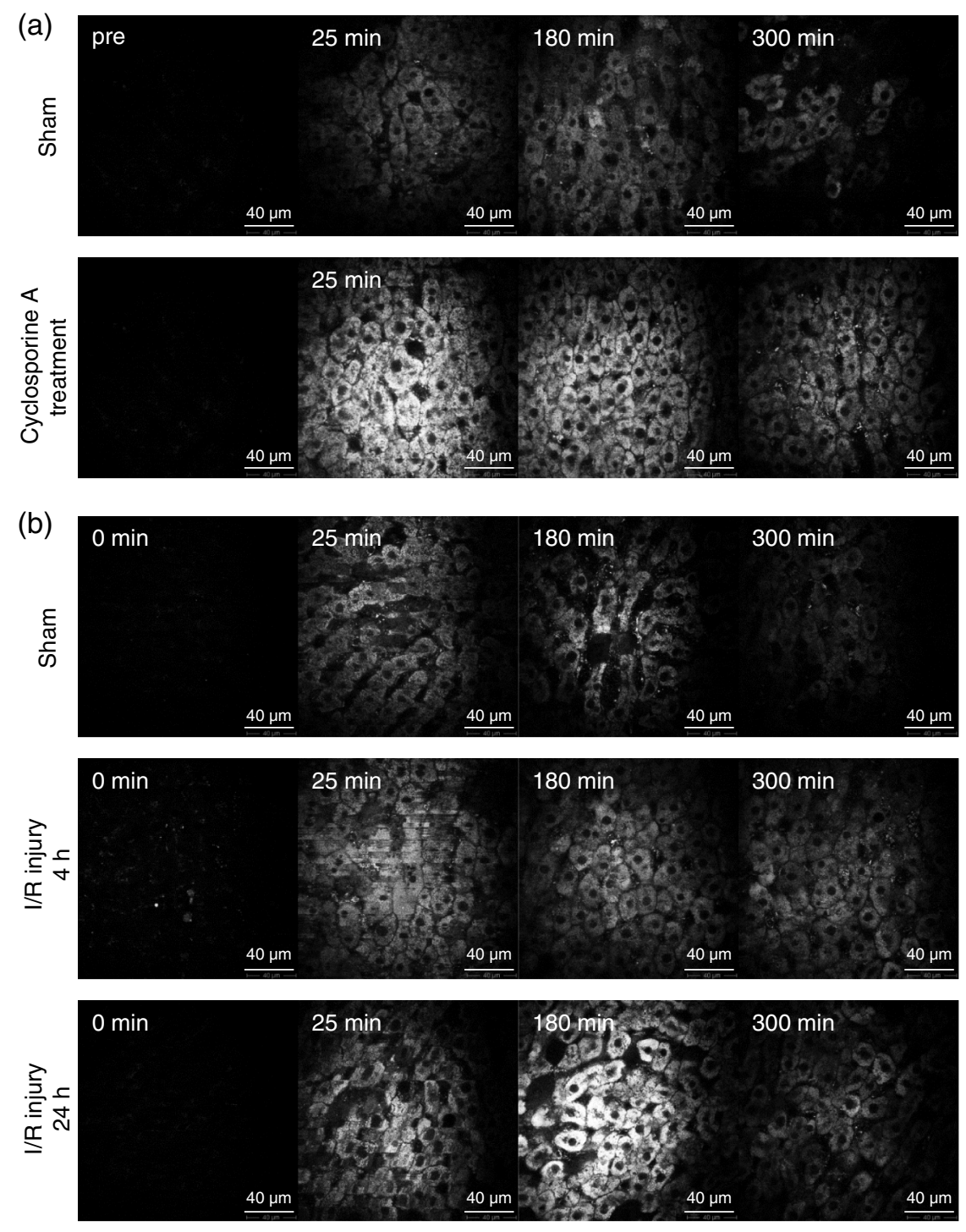

Fig. 6 Representative TPEF images of the distribution of Rh123 in the rat liver following (a) cyclosporine A treatment or (b) $\mathrm{l} / \mathrm{R}$ injury after 4 and $24 \mathrm{~h}$ of reperfusion. Images were recorded in vivo at $\lambda_{\text {Excitation/Emission }}$ of $900 / 350$ to $650 \mathrm{~nm}$, using $40 \times$ magnification, at various time-points following (a) cyclosporine $\mathrm{A}$ treatment or (b) I/R injury $(n=3$ to 4$) .{ }^{74}$

intestines, composed of fluorescent materials, could contribute to the intrinsic fluorescence in mice. This signal would be much brighter using visible wavelengths, demonstrating the advantage of using NIR imaging. ${ }^{76}$

The distribution of fluorescein and its metabolite was simultaneously investigated in real time. Initially, fluorescein's lifetime profile in phosphate buffered saline (PBS), bovine serum albumin (BSA), and bile was studied in vitro. The lifetime of fluorescein was 3.48, 3.08, and 3.41 ns in PBS, BSA, and bile, respectively. The lifetime of fluorescein monoglucuronide was 2.36, 2.39, and 2.30 ns in PBS, BSA, and bile, respectively. The lifetime was also measured in mixtures of known concentrations of fluorescein and fluorescein monoglucuronide in PBS. The lifetime of the mixture decreased with increasing concentration of fluorescein monoglucuronide. After intravenous injection of sodium fluorescein in vivo, we reported a significant drop in the fluorescence lifetime over time. This was indicative of an increased metabolism to fluorescein monoglurcuronide. ${ }^{73}$
Targeted nanoparticles may be used for the detection and treatment of human diseases; however, their short in vivo halflives limit their clinical utility and can lead to hepatotoxicity. ${ }^{77}$

\subsection{Nanoparticles}

Nanoparticles are taken up by phagocytosis, macropinocytosis, caveolin-mediated, clathrin-mediated, or celeolin/clathrin-independent endocytosis. Hydrophobic nanoparticles are rapidly removed by the reticuloendothelial system (RES), mainly by the liver and spleen. ${ }^{77}$ Due to their questionable clinical use and possible hepatotoxicity, it is important to study the hepatic transport of nanoparticles. One group showed the use of TPEF to visualize the delivery of Cy5-siRNA to the hepatocytes, with lipid nanoparticles as the delivery vehicle at the cellular level. The analysis of the diffusion of Cy5-siRNA into the hepatocytes was done by computation of the percentage signal strength in the region of interest over time. ${ }^{78}$

Another study reported a significant uptake of positively charged, but not negatively charged moieties. Negatively charged 
nanoparticles were found to be taken up by Kupffer cells as shown by intravital imaging. Positively charged nanoparticles were taken up by hepatocytes and have the potential to be cleared via hepatobiliary excretion; however, negatively charged nanoparticles accumulation in the Kupffer cell portends significant hepatotoxicity. ${ }^{77}$

Encapsulation of active substances within nanoscale particles can enhance bioavailability and biocompatibility resulting in targeted drug delivery, solubility and bioactivity, and reducing toxicity. A multimodal nonlinear label-free imaging technique to pinpoint polymeric nanoparticles within the intestine and liver was presented, where TPEF showed cellular structures, SHG collagen, and CARS generated chemical-specific images of polymer-based nanoparticles with contrast derived from the molecular vibration of carbon-deuterium bonds within the polymer's palmitic acid chains. These modalities combined to give an accurate location of the nanoparticles in relation to the cellular structure in the liver, gall bladder, and intestine. ${ }^{79}$

Quantum dots (QD) is a new class of nanomaterials recognized as a novel promising diagnostic and imaging agent. QD based on semiconductor materials are most effective for applications in the field of bioimaging, since they have unique optical and photophysical properties. QD sized 2 to $10 \mathrm{~nm}$ have high quantum yield, high brightness, and can be excited with a broad range of energies and provide strong fluorescence at visible and NIR wavelengths. In addition, they are stable and retain their bright fluorescence for a long period of time compared to organic dyes. Potential accumulation and toxicity can originate from surface charge, chemical composition, size, shape, aggregation, and type of surface coating. QD remained in the organs of the RES for over 22 days after injection, suggesting that they were consumed by mononuclear phagocytes. ${ }^{80} \mathrm{QD}$ are less prone to photobleaching and reduce the confounding influence of tissue autofluorescence and, thus, are particularly well suited for the detection of low abundance antigens, such as HCV. HCV is the leading infectious cause of cirrhosis and liver cancer in many societies. TPEF in combination with QD was used to visualize the extent and distribution of HCV infected cells within the liver. Background autofluorescence is markedly reduced in TPEF images because of the much greater TPEF action cross-sections of QD compared with endogenous tissue fluorophores. ${ }^{81}$

Imaging based on fluorescence intensity is often used to localize cellular sites where the ligand binds to its cognate receptor, but these techniques may be biased by unspecific binding and distribution of the ligand in various subcellular compartments. FLIM is sensitive to the physiochemical environment, posing an advantage over fluorescence intensity measurements. A ratiometric FLIM (rmFLIM) was used to analyze the fate of a polymer-based nanocarrier for drug delivery in the metabolic clearance process. An rmFLIM relies on the specific multiexponential lifetime signature of a protein-ligand complex that is unique compared to the fluorescence lifetime distribution of the background. Only one fluorophore is needed. ${ }^{82}$

\section{Future Directions}

A restriction of MPM is that it can only image the liver after surgery. Radiographic studies, such as abdominal ultrasonography, computed tomography, and magnetic resonance imaging, are noninvasive, but are mainly used to detect ascites, hepatosplenomegaly, hepatic or portal vein thrombosis, and hepatic carcinoma. However, the capability of detecting morphological changes associated with cirrhosis is limited. ${ }^{83}$ Microendoscopy is, therefore, promising by combining intravital microscopy and miniature endoscopy ${ }^{84}$ to conduct MPM internally in vivo safely and effectively. It can enter the body through a natural orifice or small surgical incision and could be used as a replacement or guide for biopsies. Another promising instrument is a miniaturized laser scanning microscope, which allows for minimal invasive imaging of internal organs through keyhole incisions. ${ }^{85}$ Another future aspect would be to assess liver function during surgery to establish a correct dose regimen for the individual patient. If these techniques can be translated into clinical use to assess liver function and other parts of the body, it would greatly improve early diagnosis of organ viability, fibrosis, and cancer.

\section{Acknowledgments}

We thank National Health and Medical Research Council (NHMRC \#569710) for funding this project. Also, thank you to Dr. Zhen Song for expert advice regarding physics of microscopy.

\section{References}

1. K. W. Eliceiri and C. Rueden, "Tools for visualizing multidimensional images from living specimens," Photochem. Photobiol. 81(5), 11161122 (2005).

2. N. J. Emptage, "Fluorescent imaging in living systems," Curr. Opin. Pharmacol. 1(5), 521-525 (2001).

3. D. J. Stephens and V. J. Allan, "Light microscopy techniques for live cell imaging," Science 300(5616), 82-86 (2003).

4. A. L. Grafflin and E. H. Bagley, "Studies of hepatic structure and function by fluorescence microscopy," Bull. Johns Hopkins Hosp. 90(6), 395-437 (1952).

5. I. A. Sherman, S. C. Pappas, and M. M. Fisher, "Hepatic microvascular changes associated with development of liver fibrosis and cirrhosis," Am. J. Physiol. 258(2 Pt 2), H460-465 (1990).

6. H. Popper, "Distribution of vitamin A in tissue as visualized by fluorescence microscopy," Physiol. Rev. 24(2), 205-224 (1944).

7. B. W. Volk, "Fat distribution in the human liver as revealed by fluorescence microscopy," Am. J. Dig. Dis. 17(12), 394-397 (1950).

8. V. Hanzon, "Liver cell secretion under normal and pathologic conditions studied by fluorescence microscopy on living rats," Acta Physiol. Scand. Suppl. 28(101), 1-268 (1952).

9. I. A. Sherman and M. M. Fisher, "Hepatic transport of fluorescent molecules: in vivo studies using intravital TV microscopy," Hepatology 6(3), 444-449 (1986).

10. I. Braakman, G. M. Groothuis, and D. K. Meijer, "Acinar redistribution and heterogeneity in transport of the organic cation rhodamine B in rat liver," Hepatology 7(5), 849-855 (1987).

11. P. Recknagel et al., "In vivo imaging of hepatic excretory function in the rat by fluorescence microscopy," J. Biophotonics 5(7), 571-581 (2012).

12. G. E. Stutzmann and I. Parker, "Dynamic multiphoton imaging: a live view from cells to systems," Physiol. (Bethesda) 20(1), 15-21 (2005).

13. B. R. Masters and P. T. So, "Confocal microscopy and multi-photon excitation microscopy of human skin in vivo," Opt. Express 8(1), 2-10 (2001).

14. E. E. Hoover and J. A. Squier, "Advances in multiphoton microscopy technology," Nat. Photonics 7(2), 93-101 (2013).

15. R. A. Niesner, V. Andresen, and M. Gunzer, "Intravital two-photon microscopy: focus on speed and time resolved imaging modalities," Immunol. Rev. 221(1), 7-25 (2008).

16. L. Yan et al., "Applications of combined spectral lifetime microscopy for biology," Biotechniques 41(3), 249, 251, 253 passim (2006).

17. W. Becker, "Fluorescence lifetime imaging-techniques and applications," J. Microsc. 247(2), 119-136 (2012).

18. M. S. Roberts et al., "Non-invasive imaging of skin physiology and percutaneous penetration using fluorescence spectral and lifetime imaging with multiphoton and confocal microscopy," Eur. J. Pharm. Biopharm. 77(3), 469-488 (2011).

19. M. Honda et al., "Intravital imaging of neutrophil recruitment in hepatic ischemia-reperfusion injury in mice," Transplantation 95(4), 551-558 (2013). 
20. Q. Liu et al., "Role of inducible nitric oxide synthase in mitochondrial depolarization and graft injury after transplantation of fatty livers," Free Radic. Biol. Med. 53(2), 250-259 (2012).

21. C. A. Thorling et al., "Multiphoton microscopy can visualize zonal damage and decreased cellular metabolic activity in hepatic ischemia-reperfusion injury in rats," J. Biomed. Opt. 16(11), 116011 (2011).

22. F. C. Li et al., "Investigating the effects of Panadol on mouse liver by in vivo multiphoton microscopy," Proc. SPIE 6860, 68601Y (2008).

23. F. Li et al., "Apical membrane rupture and backward bile flooding in acetaminophen-induced hepatocyte necrosis," Cell Death Dis. 2(7), e183 (2011).

24. H. Rehman et al., "Ischemic preconditioning prevents free radical production and mitochondrial depolarization in small-for-size rat liver grafts," Transplantation 85(9), 1322-1331 (2008).

25. H. Rehman et al., "NIM811 (N-methyl-4-isoleucine cyclosporine), a mitochondrial permeability transition inhibitor, attenuates cholestatic liver injury but not fibrosis in mice," J. Pharmacol. Exp. Ther. 327(3), 699-706 (2008).

26. Z. Zhong et al., "Activation of the oxygen-sensing signal cascade prevents mitochondrial injury after mouse liver ischemia-reperfusion," Am. J. Physiol. Gastrointest. Liver Physiol. 295(4), G823-G832 (2008).

27. T. Theruvath et al., " $\mathrm{C}$-Jun $\mathrm{N}$-terminal kinase 2 promotes graft injury via the mitochondrial permeability transition after mouse liver transplantation," Am. J. Transplant. 8(9), 1819-1828 (2008).

28. T. P. Theruvath et al., "Mitochondrial permeability transition in liver ischemia and reperfusion: role of c-Jun N-terminal kinase 2," Transplantation 85(10), 1500 (2008).

29. J. H. Wang et al., "Autophagy suppresses age-dependent ischemia and reperfusion injury in livers of mice," Gastroenterology 141(6), 21882199 (2011).

30. P. C. Waldmeier et al., "Inhibition of the mitochondrial permeability transition by the nonimmunosuppressive cyclosporin derivative NIM811," Mol. Pharmacol. 62(1), 22-29 (2002).

31. H. Rehman et al., "NIM811 prevents mitochondrial dysfunction, attenuates liver injury and stimulates liver regeneration after massive hepatectomy," Transplantation 91(4), 406-412 (2011).

32. C. A. Thorling et al., "Intravital multiphoton microscopy can model uptake and excretion of fluorescein in hepatic ischemia-reperfusion injury," J. Biomed. Opt. 18(10), 101306 (2013).

33. S.-F. Wang et al., "7-Ketocholesterol induces P-glycoprotein through $\mathrm{PI} 3 \mathrm{~K} / \mathrm{mTOR}$ signaling in hepatoma cells," Biochem. Pharmacol. 86(4), 548-560 (2013).

34. D. C. Tai et al., "Fibro-C-Index: comprehensive, morphology-based quantification of liver fibrosis using second harmonic generation and two-photon microscopy," J. Biomed. Opt. 14(4), 044013 (2009).

35. L. Gailhouste et al., "Fibrillar collagen scoring by second harmonic microscopy: a new tool in the assessment of liver fibrosis," J. Hepatol. 52(3), 398-406 (2010).

36. W. Sun et al., "Nonlinear optical microscopy: use of second harmonic generation and two-photon microscopy for automated quantitative liver fibrosis studies," J. Biomed. Opt. 13(6), 064010 (2008).

37. J. H. Lee et al., "Rapid diagnosis of liver fibrosis using multimodal multiphoton nonlinear optical microspectroscopy imaging," J. Biomed. Opt. 18(7), 076009 (2013).

38. Y.-M. Wu et al., "Quantitative assessment of hepatic fat of intact liver tissues with coherent anti-Stokes Raman scattering microscopy," Anal. Chem. 81(4), 1496-1504 (2009).

39. Y. He et al., "Toward surface quantification of liver fibrosis progression," J. Biomed. Opt. 15(5), 056007 (2010).

40. J. Lin et al., "An integrated coherent anti-stokes Raman scattering and multiphoton imaging technique for liver disease diagnosis," Proc. SPIE 8226, 822625 (2012).

41. W. R. Zipfel, R. M. Williams, and W. W. Webb, "Nonlinear magic: multiphoton microscopy in the biosciences," Nat. Biotechnol. 21(11), 1369-1377 (2003).

42. M. Goetz et al., "In vivo confocal laser endomicroscopy of the human liver: a novel method for assessing liver microarchitecture in real time," Endoscopy 40(07), 554-562 (2008).

43. M. Goetz et al., "In vivo confocal laser laparoscopy allows real time subsurface microscopy in animal models of liver disease," J. Hepatol. 48(1), 91-97 (2008).
44. J. Lin et al., "Assessment of liver steatosis and fibrosis in rats using integrated coherent anti-Stokes Raman scattering and multiphoton imaging technique," J. Biomed. Opt. 16(11), 116024 (2011).

45. H.-S. Lee et al., "Optical biopsy of liver fibrosis by use of multiphoton microscopy," Opt. Lett. 29(22), 2614-2616 (2004).

46. Y. Liu et al., "Multiphoton analysis of normal and diseased livers," Proc. SPIE 5700, 249-255 (2005).

47. M. S. Roberts et al., "In vitro and in vivo imaging of xenobiotic transport in human skin and in the rat liver," J. Biophotonics 1(6), 478-493 (2008).

48. T. Guilbert et al., "A robust collagen scoring method for human liver fibrosis by second harmonic microscopy," Opt. Express 18(25), 25794-25807 (2010).

49. J.-W. Jhan et al., "Integrated multiple multi-photon imaging and Raman spectroscopy for characterizing structure-constituent correlation of tissues," Opt. Express 16(21), 16431-16441 (2008).

50. A. Zumbusch, G. R. Holtom, and X. S. Xie, "Three-dimensional vibrational imaging by coherent anti-Stokes Raman scattering," Phys. Rev. Lett. 82(20), 4142-4145 (1999).

51. T.-L. Sun et al., "Ex vivo imaging and quantification of liver fibrosis using second-harmonic generation microscopy," J. Biomed. Opt. 15(3), 036002 (2010).

52. T.-L. Sun et al., "Human hepatocellular carcinoma diagnosis by multiphoton autofluorescence microscopy," Proc. SPIE 7555, 75551L (2010).

53. J. Yan et al., "Use of multiphoton microscopy to diagnose liver cancer and lung metastasis in an orthotopic rat model," Scanning 34(4), 271277 (2012).

54. Y. Liu et al., "Multiphoton autofluorescence and second-harmonic generation diagnosis of hepatocellular carcinoma," Proc. SPIE 6863, 68630J (2008).

55. T. Takeichi et al., "4-dimensional intravital microscopy: a new model for studies of leukocyte recruitment and migration in hepatocellular cancer in mice," J. Gastrointest. Surg. 14(5), 867-872 (2010).

56. T.-M. Liu et al., "Diagnosing hepatocellular carcinoma with the intensity and the lifetime of two-photon red autofluorescences," Proc. SPIE 7903, $79032 Z$ (2011).

57. T.-L. Sun et al., "Label-free diagnosis of human hepatocellular carcinoma by multiphoton autofluorescence microscopy," Appl. Phys. Lett. 95(19), 193703 (2009).

58. E. A. te Velde et al., "Early endostatin treatment inhibits metastatic seeding of murine colorectal cancer cells in the liver and their adhesion to endothelial cells," Br. J. Cancer 92(4), 729-735 (2005).

59. S. Bloch et al., "Whole-body fluorescence lifetime imaging of a tumortargeted near-infrared molecular probe in mice," J. Biomed. Opt. 10(5), 054003 (2005).

60. F.-C. Li et al., "In vivo dynamic metabolic imaging of obstructive cholestasis in mice," Am. J. Physiol. Gastrointest. Liver Physiol. 296(5), G1091-G1097 (2009).

61. M. S. Roberts et al., "Enterohepatic circulation," Clin. Pharmacokinet. 41(10), 751-790 (2002)

62. S. Elmore, "Apoptosis: a review of programmed cell death," Toxicol. Pathol. 35(4), 495-516 (2007).

63. A. M. Seifalian et al., "Effects of hepatic ischaemia/reperfusion injury in a rabbit model of indocyanine green clearance," Clin. Sci. 102(5), 579-586 (2002).

64. M. Mendes-Braz et al., "The current state of knowledge of hepatic ischemia-reperfusion injury based on its study in experimental models," J. Biomed. Biotechnol. 2012, 1-20 (2012).

65. R. Barhoumi et al., "Analysis of benzo [a] pyrene partitioning and cellular homeostasis in a rat liver cell line," Toxicol. Sci. 53(2), 264-270 (2000).

66. R. Barhoumi et al., "Multiphoton spectral analysis of benzo [a] pyrene uptake and metabolism in a rat liver cell line," Toxicol. Appl. Pharmacol. 253(1), 45-56 (2011).

67. D. Svistounov et al., "The relationship between fenestrations, sieve plates and rafts in liver sinusoidal endothelial cells," PloS One 7(9), e46134 (2012).

68. M. Weiss et al., "Functional characterization of hepatic transporters using intravital microscopy," Eur. J. Pharm. Sci. 49(5), 845-849 (2013).

69. Y. Liu et al., "Visualization of hepatobiliary excretory function by intravital multiphoton microscopy," J. Biomed. Opt. 12(1), 014014 (2007). 
70. F.-C. Li et al., "Intravital multiphoton microscopy for imaging hepatobiliary function," Proc. SPIE 6630, 663014 (2007).

71. W. Lo et al., "Intravital multiphoton microscopy for imaging hepatobiliary function," Proc. SPIE 6442, 64421R (2007).

72. Y. Liu et al., "In vivo multiphoton imaging of bile duct ligation," Proc. SPIE 6860, 68600G (2008).

73. C. A. Thorling et al., "Multiphoton microscopy and fluorescence lifetime imaging provide a novel method in studying drug distribution and metabolism in the rat liver in vivo," J. Biomed. Opt. 16(8), 086013 (2011).

74. X. Liu et al., "Intravital multiphoton imaging of rhodamine 123 in the rat liver after intravenous dosing," IntraVital 1(1), 54-59 (2012).

75. R. Parasrampuria, I. H. Shaik, and R. Mehvar, "Effects of in vivo hepatic ischemia-reperfusion injury on the hepatobiliary disposition of rhodamine 123 and its metabolites in isolated perfused rat livers," J. Pharm. Pharm. Sci. 15(2), 318-328 (2012).

76. W. Akers et al., "In vivo resolution of multiexponential decays of multiple near-infrared molecular probes by fluorescence lifetime-gated whole-body time-resolved diffuse optical imaging," Mol. Imaging 6(4), 237-246 (2007).

77. S.-H. Cheng et al., "Visualizing dynamics of sub-hepatic distribution of nanoparticles using intravital multiphoton fluorescence microscopy," ACS Nano 6(5), 4122-4131 (2012).

78. A. Chen et al., "Quantification of Cy-5 siRNA signal in the intra-vital multi-photon microscopy images," in Annual Int. Conf. of the IEEE Engineering in Medicine and Biology Society, pp. 3712-3715, IEEE, Piscataway, NJ (2012).

79. N. L. Garrett et al., "Exploring uptake mechanisms of oral nanomedicines using multimodal nonlinear optical microscopy," J. Biophotonics 5(5-6), 458-468 (2012).

80. Y. F. Loginova et al., "Biodistribution of intact fluorescent CdSe/CdS/ $\mathrm{ZnS}$ quantum dots coated by mercaptopropionic acid after intravenous injection into mice," J. Biophotonics 5(11-12), 848-859 (2012).

81. Y. Liang et al., "Visualizing hepatitis $\mathrm{C}$ virus infections in human liver by two-photon microscopy," Gastroenterology 137(4), 1448-1458 (2009).

82. A. Boreham et al., "Exploiting fluorescence lifetime plasticity in FLIM: target molecule localization in cells and tissues," ACS Med. Chem. Lett. 2(10), 724-728 (2011).

83. J.J.Heidelbaugh and M. Bruderly, "Cirrhosis and chronic liverfailure: part I. Diagnosis and evaluation," Am. Fam. Physician 74(5), 756-762 (2006).
84. P. Kim et al., "In vivo confocal and multiphoton microendoscopy," J. Biomed. Opt. 13(1), 010501 (2008).

85. H. Alencar et al., "Novel multiwavelength microscopic scanner for mouse imaging," Neoplasia (New York, NY) 7(11), 977 (2005).

Camilla A. Thorling completed her PhD studies at the University of South Australia under the supervision of Professor Michael Roberts and was awarded her degree in March 2014. Her research interests include the application of multiphoton microscopy and fluorescence lifetime imaging in the progression of liver disease.

Darrell Crawford is the head of the School of Medicine. He graduated from the UQ School of Medicine and completed his advanced training in gastroenterology in Brisbane. He trained in liver disease with Professor Lawrie Powell and was awarded his MD in 1994.

Frank J. Burczynski is working at the Department of Pharmacology and Therapeutics, Faculty of Pharmacy, University of Manitoba, Canada. His research focus is on cellular drug uptake mechanisms in normal and diseased livers.

Xin Liu received her PhD from the National University of Singapore. Her research interests are drug pharmacokinetic modeling and the application of multiphoton microscopy in liver disease.

Ian Liau received his $\mathrm{PhD}$ degree in physical chemistry from the University of Chicago. He joined Fluidigm Corporation (USA) engaging in the development of microfluidic technology. He was later employed as a researcher at the Biomedical Engineering Center, leading a team to develop medical devices. He joined National Chiao Tung University in 2005 and has been an associate professor since 2010. His research focuses on biomedical applications of optical imaging and spectroscopy.

Michael S. Roberts is an NHMRC senior principal research fellow and director at the Therapeutics Research Centre, School of Medicine at the University of Queensland, and at the School of Pharmacy and Medical Sciences at the University of South Australia. His key research areas of interest are rational drug design, transdermal drug delivery, pharmacokinetics, and quality use of medicines. 